\title{
Respostas evocadas de incongruência a categorias na percepção da fala*
}

\author{
Mismatch evoked responses to categories in speech perception \\ Daniel Márcio Rodrigues Silva \\ Rui Rothe-Neves \\ Universidade Federal de Minas Gerais - Belo Horizonte - Minas Gerais - Brasil
}

$\diamond$

\begin{abstract}
Resumo: A percepção dos sons da fala envolve operações de categorização em que os eventos acústicos que compõem o fluxo sonoro da fala são agrupados em classes de equivalência, ou seja, categorias cujos membros são tratados pelos falantes como funcionalmente equivalentes. Diversas linhas de evidência indicam efeitos relacionados a essas categorias sobre a percepção. Neste contexto, medidas da atividade neuronal relacionada ao processamento perceptivo no córtex cerebral são de grande relevância. Estudos psicofisiológicos indicam que o componente das respostas evocadas auditivas denominado "negatividade de incongruência" (MMN, ou Mismatch Negativity), considerado como um índice da memória sensorial auditiva e da discriminação entre estímulos sonoros, é sensível não apenas aos atributos físicos dos sons da fala, mas também ao modo como os mesmos são categorizados pelos falantes de uma língua. No presente trabalho é apresentada uma retrospectiva crítica sobre o emprego dessa resposta nas investigações sobre a categorização dos sons da fala e uma apreciação de suas contribuições para a compreensão do aspecto perceptivo da comunicação verbal.
\end{abstract}

Palavras-chave: Negatividade de incongruência (MMN); Potenciais evocados; Campos magnéticos evocados; Percepção da fala; Categorização

\begin{abstract}
Speech perception involves categorization operations in which acoustic events that compose the speech stream are grouped into equivalence classes, i. e., categories whose members are treated by speakers as functionally equivalent. Several lines of evidence indicate effects of speech sound categories on perception. In this context, measures of neural activity related to perceptual processing at the level of the cerebral cortex are of great relevance. Psychophysiological studies show that the Mismatch Negativity (MMN) component of the auditory evoked responses, which has been regarded as an index of auditory sensory memory and discrimination, is sensitive not only to the physical attributes of the speech sounds but also to the way those sounds are categorized by the speakers of a language. The present work offers a critical retrospective review of the use of the MMN response in research on speech sound categorization and an appreciation of its contributions to the comprehension of the perceptual aspects of speech communication.
\end{abstract}

Keywords: Mismatch negativity (MMN); Evoked potentials; Evoked fields; Speech perception; Categorization

\section{Introdução}

No tema da interface entre Fonética e Fonologia, um dos tópicos mais produtivos é o debate entre o papel que cabe à representação fonética e à representação fonológica, discussão frequentemente subsumida sob aquela dos traços distintivos. Este é o assunto do presente artigo, em que ambas são consideradas a partir do ponto de vista da percepção da fala, ao contrário do que é usual na área. Na visão clássica exposta por Chomsky e Halle (1968, doravante SPE), compreendem-se por Fonologia as regras e representações discretas de uma dada língua, enquanto à Fonética cabe a implementação mecânica da Fonologia. Não se diz ali o que é nem como ocorre a percepção da fala, mesmo que o primeiro modelo a tratar do fenômeno lhe seja anterior, a famosa teoria motora

\footnotetext{
* O presente trabalho foi realizado com o apoio da Coordenação de Aperfeiçoamento de Pessoal de Nível Superior - CAPES - Brasil.
} 
em sua primeira versão (LIBERMAN et al., 1967). Na herança do SPE, a percepção da fala seria externa à gramática fonológica e relegada à Psicolinguística até muito depois (ver p. ex. Klatt, 1989).

No quadro da Teoria da Otimidade, formalismo em que os padrões fônicos são caracterizados em termos de conflitos entre restrições universais foneticamente fundamentadas, a percepção da fala pôde ter um lugar constituinte na gramática fonológica, um movimento iniciado logo depois da obra seminal de Prince e Smolensky (1993). São exemplos disso a Teoria da Dispersão (FLEMMING, 2002), a Teoria da Dispersão Adaptativa (PADGETT, 1997), a abordagem ao enfraquecimento de consoantes baseada no esforço (KIRCHNER, 1998) e a Fonologia Funcional (BOERSMA, 1998), depois renomeada como Fonologia Bidirecional (BOERSMA; HAMANN, 2008; 2009). Comum a todos esses modelos é a tentativa de formalizar uma teoria fonológica em que não se supõe uma diferença qualitativa da Fonética para a Fonologia: a percepção da fala é concebida na gramática fonológica como o conjunto de restrições que se aplicam diretamente sobre o substrato fonético a fim de abstrair as representações fonéticas ou de superfície (FLEMMING, 2008).

Nesse empreendimento, o papel que cabe à representação fonética eventualmente terminaria por se confundir com aquele que se atribui à representação fonológica. Tradicionalmente, representação fonética é o resultado do processo de extração daquelas características importantes que permitem ao falante ouvir apenas $[\mathrm{z}]$ quando de fato a onda acústica que lhe afetou os tímpanos carregava diversas outras informações - sobre o sexo ou a idade do seu interlocutor, sobre as vogais ou consonantes que ladeavam a fricativa etc. Representação fonológica, por outro lado, é o resultado da aplicação das regras fonológicas ou - menos tradicionalmente - da verificação comparada às restrições, resultando apenas na informação sobre sua natureza categórica, distintiva ou não. Parece, portanto, que os níveis de representação fonética e fonológica têm estatutos diferentes. A questão empírica que se coloca é: a qual nível de abstração a partir da onda acústica podemos atribuir as representações fônicas?

Neste artigo, vamos discutir essa questão a partir de um ponto de vista empírico muito particular: aquele de dentro do cérebro humano. Afinal, se a percepção da fala, por definição, é um processo que não se observa diretamente, melhor será utilizar técnicas específicas para concluir sobre suas características. Aqui, a técnica de escolha é a das respostas evocadas auditivas ${ }^{1}$. Ao ser exposto repetidamente a estímulos sonoros, o

\footnotetext{
Há várias técnicas para avaliação objetiva da modificação do funcionamento cerebral a partir da apresentação de diferentes estímulos linguísticos, sobre as quais o leitor encontrará uma revisão em Rothe-Neves (2001).
}

cérebro humano produz ondas elétricas que podem ser investigadas a fim de se conhecer quais características são percebidas e discriminadas no córtex cerebral O objetivo é compreender quais evidências já foram produzidas que nos permitam iluminar a questão da representação fônica. Com isso, atendemos ao requisito de compatibilidade externa (BUNGE, 1976), pelo qual uma teoria não deve ser incompatível com o conhecimento produzido em outras áreas.

\section{A Percepção da fala cérebro adentro}

Respostas à estimulação auditiva obtidas de grandes populações de neurônios no encéfalo podem ser medidas no couro cabeludo por eletrodos sensíveis a potenciais elétricos, no caso da eletroencefalografia, ou sensores capazes de detectar campos magnéticos, no caso da magnetoencefalografia. Trata-se, respectivamente, dos denominados potenciais evocados e campos magnéticos evocados auditivos (STEINSCHNEIDER; LIÉGEOISCHAUVEL; BRUGGE; 2011; LÜTKENHÖNER; POEPPEL, 2011). Se um som é apresentado repetidamente a uma pessoa enquanto se registra seu eletroencefalograma (EEG), uma curva representando a média coerente dos trechos de atividade registrada durante cada prova apresenta normalmente três declinações com picos por volta de 50, 100 e $200 \mathrm{~ms}$ após o início do estímulo. A primeira e a terceira dessas variações têm polaridades positivas e a segunda, polaridade negativa (Fig. 1). São os componentes P1, N1 e P2 da resposta evocada de longa latência. Componentes análogos ocorrem em sinais magnetoencefalográficos e serão aqui designados de acordo com a convenção de se adicionar a letra ' $m$ ' seguindo o nome do componente, ou seja, P1m, N1m e P2m (para revisões sobre os potenciais e campos evocados auditivos, veja STEINSCHNEIDER et al., 2011; LÜTKENHÖNER; POEPPEL, 2011).

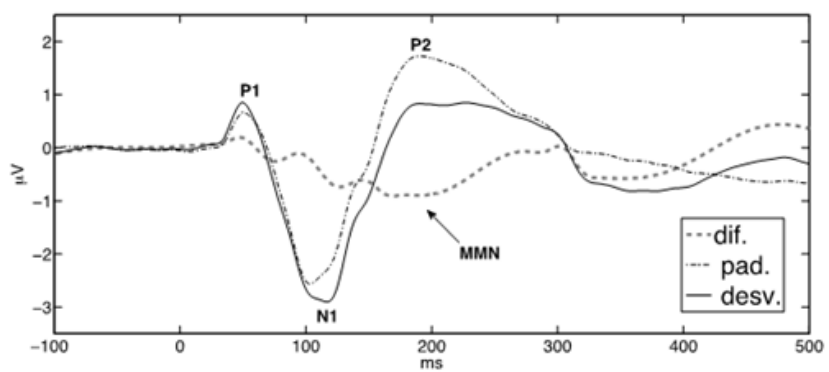

Figura 1 - Potenciais evocados fictícios ilustrando componentes de respostas típicas aos estímulos padrão (pad.) e desviante (desv.) em um protocolo de estimulação tipo oddball. Uma operação de subtração entre as duas respostas resulta em uma "onda de diferença" (dif.=desv.-pad), na qual o componente MMN aparece como uma deflexão negativa. 
Em uma técnica amplamente utilizada, uma pequena parte dos sons (por exemplo, 10\%) em meio à sequência repetitiva de estimulação pode ser trocada por outros que sejam diferentes dos primeiros em um parâmetro qualquer, de modo a se utilizar uma sequência com um estímulo mais frequente, denominado padrão, e um mais raro, o desviante. Esse paradigma experimental, conhecido como oddball, permite investigar respostas relacionadas à capacidade e ao processo de discriminação entre os estímulos empregados como padrão e desviante. Quando a onda de EEG correspondente ao padrão é subtraída da onda que representa a resposta ao estímulo desviante, obtém-se normalmente uma "onda de diferença" caracterizada por uma negatividade de incongruência (Mismatch Negativity, doravante MMN; vide Fig. 1) uma deflexão negativa com pico em geral entre 150 e 250 ms após o início do desvio (NÄÄTÄNEN et al., 2007). Assim como os componentes P1, N1 e P2, a MMN é uma resposta automática, ou "pré-atencional", pois sua ocorrência não depende de que o foco da atenção esteja sobre o estímulo que a provoca.

De acordo com a explicação predominante (NÄÄTÄNEN; KUJALA; WINKLER, 2011;NÄÄTÄNEN; WINKLER, 1999; NÄÄTÄNEN, 1990), a MMN reflete um processo de detecção de mudanças em que a informação da entrada sensorial em um dado momento é comparada a um vestígio na memória sensorial. Esse vestígio representa as regularidades que caracterizam a sequência repetitiva de estímulos estabelecendo previsões sobre a estimulação subsequente. Isto é, mecanismos neuronais relacionados a esse processo seriam ativados por violações dessas previsões, produzindo uma resposta que se traduz no potencial evocado como a MMN. A formação de tal vestígio sensorial dependeria não apenas da entrada estimulatória, mas também de vestígios de memória de logo prazo, o que explica o efeito da experiência/ aprendizagem sobre esse componente (de que trataremos mais adiante). Nessa linha, e com base em uma série de evidências experimentais, Näätänen e Winkler (1999) propõem a MMN como um índice do que designam como "representação central do som" - uma representação unitária do evento auditivo, resultado do processamento paralelo e integração dos atributos acústico-sensoriais do estímulo, e que corresponde ao percepto que pode se apresentar à consciência ${ }^{2}$.

\footnotetext{
2 Há explicações alternativas, incluindo a proposta de que a MMN seja resultado de modulações na latência e na amplitude do componente N1 da resposta evocada pelo estímulo desviante (JÄÄSKELÄINEN et al., 2004; MAY; TIITINEN, 2010). Propostas conciliatórias também foram apresentadas com base em evidências de neuroimagem (LAUFER; NEGISHI; CONSTABLE, 2009; LAUFER et al., 2008; MAESS et al., 2007; OPITZ et al., 2005) e simulações computacionais (GARAGNANI; PULVERMÜLLER, 2011)
}

\subsection{Mapas "fonemotópicos" no córtex auditivo}

Uma série de estudos magnetoencefalográficos encontram evidências de um mapeamento dos sons da fala no nível do córtex auditivo em que sons distintos são codificados por padrões espaciais distintos de atividade neuronal. Foram observadas, por exemplo, diferenças na localização da atividade geradora do componente N1m em função de distinções entre vogais (DIESCH et al., 1996; MÄKELÄ et al., 2004; OBLESER, ELBERT et al., 2003; OBLESER; LAHIRI; EULITZ, 2004; SCHARINGER; IDSARDI; POE, 2011; SHESTAKOVA et al., 2004) e entre consoantes (OBLESER; LAHIRI; EULITZ, 2003; OBLESER; SCOTT; EULITZ, 2006). Scharinger, Idsardi e Poe (2011) propõem um modelo de mapeamento em que vogais recuadas são mapeadas no córtex auditivo sobre um plano definido pelos eixos anatômicos latero-lateral e antero-posterior, e as vogais anteriores sobre um plano definido pelos eixos latero-lateral e longitudinal (inferiorsuperior). Uma questão que se coloca é a do nível de abstração das representações que se manifestam nesses resultados: tratam-se de representações compostas apenas por informação sensorial referente a parâmetros acústicos dos estímulos ou, alternativamente, de representações discretas, mais abstratas, talvez baseadas em traços distintivos dos sons da fala?

Scharinger, Idsardi e Poe (2011) mostram que seus dados se ajustam melhor a um modelo baseado em traços distintivos de altura, ponto de articulação e arredondamento do que a um modelo baseado em parâmetros acústicos contínuos (no caso, as frequências dos três primeiros formantes da vogal; doravante, F1, F2 e F3). Entretanto, a conclusão de que a localização da resposta $\mathrm{N} 1 \mathrm{~m}$ reflete representações baseadas em traços distintivos não é segura, uma vez que os valores de F1, F2 e F3 das vogais utilizadas como estímulos formam agrupamentos de acordo com os mesmos traços. Resultados recentes sugerem que a MMN em respostas evocadas a vogais é especialmente sensível aos picos espectrais correspondentes a ressonâncias acústicas do trato vocal, ou seja, aos formantes (TUOMAINEN et al., 2013).

\subsection{Manifestações neurofisiológicas das categorias fônicas}

Questões relacionadas aos níveis de abstração das representações dos sons da fala e seu substrato neural têm sido abordadas em estudos da MMN. Resultados indicam de maneira consistente que a $\mathrm{MMN}$ em resposta a vogais ou sílabas isoladas é gerada em um ponto do processamento em que a informação fonética foi extraída em meio a 
variações linguisticamente irrelevantes na frequência fundamental (doravante, $\mathrm{F}_{0}$; AULANKO; et al., 1993; DEGUCHI et al., 2010; JACOBSEN; SCHRÖGER; ALTER, 2004), na intensidade (JACOBSEN et al., 2004) e em atributos relacionados à voz do falante (SHESTAKOVA et al., 2002). Sendo a MMN um componente associado à detecção de um estímulo desviante, verifica-se que esta resposta ocorre mesmo em condições experimentais em que os estímulos desviante e padrão variam livremente em parâmetros acústicos irrelevantes à distinção fonética entre os dois (por exemplo, a distinção entre as sílabas / bæ/ e /gæ/ em AULANKO et al., 1993). Esses achados podem ser interpretados como evidências de que, em um nível pré-atencional do processamento perceptivo, sons acusticamente diferentes são agrupados em categorias fônicas e identificados de acordo com as mesmas.

$O$ fato de que a categorização dos sons da fala depende da experiência linguística do falante se reflete nas respostas evocadas auditivas na forma de efeitos sobre a MMN causados por categorias fônicas específicas à língua. Em um experimento com falantes do francês, Dehaene-Lambertz (1997) obteve potenciais evocados em resposta a um estímulo desviante identificado como um exemplar da sílaba /ba/, precedido por três apresentações de um estímulo padrão, que ora era outra variante da sílaba /ba/, ora um exemplar da sílaba /da/. A MMN foi observada apenas no segundo caso, isto é, quando as consoantes nos estímulos padrão e desviante pertenciam a categorias diferentes - mesmo sendo equivalentes as diferenças acústicas entre os dois desviantes e o estímulo padrão. Nenhuma MMN foi observada numa condição em que o estímulo desviante era uma sílaba [da] e o estímulo padrão, uma sílaba [da], cuja consoante é uma oclusiva retroflexa empregada no hindi, mas identificada por falantes do francês como uma variante atípica da consoante /d/. Sharma e Dorman (1999) apresentaram a falantes do inglês sequências oddball em que os estímulos padrão e desviante consistiam em sílabas que diferiam (sempre por $30 \mathrm{~ms}$ ) no tempo de início de vozeamento (voice onset time, VOT). Verificaram uma MMN robusta quando o estímulo padrão era uma sílaba identificada como /da/ e o desviante uma sílaba identificada como /ta/, mas uma MMN diminuta quando ambos eram identificados como /ta/. Em estudo posterior (SHARMA; DORMAN, 2000), os estímulos padrão e desviante eram ambos identificados como variantes da sílaba /ba/ por falantes do inglês, mas como $/ \mathrm{pa} / \mathrm{e} / \mathrm{ba} /$ por falantes do hindi. Apenas entre os últimos foi detectada a MMN. De acordo com estes resultados, a MMN parece estar associada não a diferenças acústicas entre os estímulos padrão e desviante, mas à distinção entre categorias fônicas a partir das quais os falantes identificam sons da fala.
Há, entretanto, relatos da ocorrência da MMN associada a diferenças acústicas entre sons da fala e não a distinções entre categorias fônicas (MAISTE et al., 1995; SAVELA et al., 2003; SHARMA et al., 1993). Winkler et al. (1999) sugerem que o processo gerador da MMN opera tanto com representações categóricas específicas à língua como com representações dos eventos auditivos ricas em informação sobre parâmetros acústicos. Esses autores realizaram um estudo em que foram empregados dois pares de vogais, um dos quais entre categorias (cada vogal identificada a uma categoria vocálica diferente) em húngaro e dentro da categoria (ambas as vogais identificadas a uma mesma categoria) em finlandês, valendo o inverso para o outro par - isto é, entre categorias em finlandês e dentro da categoria em húngaro. Para cada par, uma das vogais foi apresentada como estímulo padrão e a outra como estímulo desviante. A MMN foi observada em falantes do finlandês e do húngaro para ambos os pares, mas sua amplitude depende do modo como cada língua determina a categorização das vogais, sendo maior quando os estímulos padrão e desviante pertencem a categorias diferentes - ou seja, os falantes do húngaro e do finlandês apresentaram padrões inversos entre si. Essas diferenças em amplitude indicam um efeito de categorias fônicas específicas à língua sobre a $\mathrm{MMN}$, ao passo que a ocorrência dessa resposta em todas as condições sugere que ela reflete também o processamento da informação auditiva que possibilita a discriminação entre exemplares de uma mesma categoria (veja também KIRMSE et al., 2008; NENONEN et al., 2003; ZHANG et al., 2005). Em outras palavras, uma resposta eletrofisiológica a diferenças acústicas é exagerada quando tais diferenças são usadas funcionalmente numa língua.

Esse efeito "linguístico" foi investigado especificamente por Dehaene-Lambertz et al. (2005). Os pesquisadores usaram como estímulos uma série de tons complexos que normalmente não seriam reconhecidos como sons da fala, mas podem, mediante instrução, ser identificados como exemplares distorcidos de sílabas variando entre $/ \mathrm{ba} / \mathrm{e} / \mathrm{da} /$. Efeitos dessas categorias sobre o desempenho em uma tarefa comportamental de discriminação e sobre a MMN foram observados apenas quando os participantes foram instruídos a identificar os estímulos como sílabas, resultado que vai ao encontro de estudos comportamentais que indicam que a discriminação entre sons da fala pode, de acordo com as demandas da tarefa, se dar em um modo categórico ou em um modo não-categórico em que os eventos sonoros são avaliados a partir de parâmetros psicoacústicos contínuos (GERRITS; SCHOUTEN, 2004; SCHOUTEN; GERRITS; VAN HESSEN; 2003; SILVA; ROTHE-NEVES, 2009).

Näätänen et al. (1997) observaram que a vogal /ø/, acusticamente mais próxima a um estímulo padrão /e/, 
elicia uma MMN com menor amplitude se comparada à vogal $/ \gamma /$ em falantes do estoniano, língua em que essas três vogais são usadas. Já no caso de falantes do finlandês, para os quais $/ \gamma /$ não é familiar, maiores amplitudes foram observadas na MMN em resposta à vogal $/ \varnothing /$. O mesmo foi observado em bebês finlandeses de 12 meses, mas não em bebês estonianos com a mesma idade ou bebês finlandeses de 6 meses (CHEOUR et al., 1998). Esses achados indicam que a sensibilidade da MMN à experiência linguística, atestada nos estudos supracitados, está relacionada a efeitos de familiaridade - sons mais familiares causam respostas mais robustas. Isso é coerente com a explicação de que a MMN seja resultante de uma incongruência na comparação entre a representação auditiva de um estímulo e seu vestígio na memória sensorial, influenciado pela memória de longa duração.

Contudo, outros fatores devem ser considerados. Em primeiro lugar, Tervaniemi et al. (2006) fornecem evidências de que falantes do finlandês apresentam um desempenho superior na discriminação e uma MMN mais pronunciada em resposta a mudanças na duração de tons complexos (não verbais) quando comparados a falantes do alemão. Os autores relacionam essa diferença ao fato de que distinções de duração têm maior importância na fonologia do finlandês do que na fonologia do alemão, sugerindo que a experiência linguística poderia influenciar não apenas a percepção dos sons da fala, mas o processamento auditivo de maneira geral. Por outro lado, em outro estudo cujos resultados também não se explicam por efeitos de familiaridade, Liu e Holt (2011) relataram aumentos na amplitude e redução na latência da MMN para pares entre categorias e diminuição da amplitude para pares dentro das categorias após a aprendizagem de categorias artificiais formadas por sons não-verbais, mostrando que este tipo de efeito não resulta apenas da experiência linguística, mas pode ser causado por qualquer aprendizagem de categorização.

\subsection{Representações discretas: um nível superior de abstração}

Os estudos revistos até este ponto fornecem evidências importantes do processamento perceptivo pré-atencional de informação relativa a categorias fônicas formadas a partir da experiência linguística. Note-se, porém, que a categorização dos sons da fala pode ocorrer em diferentes níveis de representação entre a entrada sensorial e a mensagem linguística. Categorias fônicas podem ser formadas a partir de padrões estatísticos de ocorrência de sons, o que se verifica já no primeiro ano de idade (CHEOUR et al., 1998; KUHL et al., 2006,
2008; WERKER; TEES, 2005)3․ Com a aquisição de um léxico, é possível postular categorias estabelecidas pala estrutura fonológica da língua em um nível mais abstrato de representação, em que, por exemplo, as duas primeiras consoantes da palavra "título", pronunciada como ['t titulu], são equivalentes, ou seja, identificadas a uma mesma categoria /t/. De acordo com Phillips (2001), trata-se de um nível de processamento simbólico, que opera com representações discretas que não incluem informação acerca de variações internas às categorias e sobre as quais não é possível obter evidências seguras por' meio de comparações entre condições experimentais em que estímulos padrão e desviantes constituem pares dentro de e entre categorias. Esse nível de processamento poderia se manifestar em condições experimentais que permitam demonstrar que toda a variabilidade dentro da categoria é definitivamente ignorada pelo sistema perceptivo.

$\mathrm{Na}$ tentativa de elaborar um delineamento experimental adequado para esse fim, Phillips et al. (2000) produziram uma série de sílabas variando em VOT, entre as categorias /dæ/ e /tæ/, e definiram um conjunto de estímulos padrão composto por sílabas variando dentro de uma das categorias e um conjunto de estímulos desviantes formado por sílabas variando dentro da outra categoria. Deste modo, a distinção entre estímulos padrão e desviante pode ser estabelecida não em termos das sílabas tomadas individualmente, mas de uma categoria que ocorre com maior frequência na sequência de estímulos e uma categoria menos frequente. Uma MMNm com forma e latência típicas em resposta às sílabas pertencentes à categoria menos frequente foi observada e interpretada como evidência de representações fonológicas discretas no córtex auditivo (região em que foi localizada a resposta). Um estudo semelhante com categorias vocálicas foi realizado por Hill, McArthur e Bishop (2004), que relatam uma deflexão negativa adicional entre 350 e 500 ms, identificada como uma "MMN tardia", associada à categoria menos frequente ${ }^{4}$.

Kazanina, Phillips e Idsardi (2006) conduziram um estudo com falantes do russo e do coreano sobre a distinção /d:t/ nas duas línguas. Na língua russa, há um contraste fonológico entre essas duas consoantes, o que não ocorre no coreano, em que são alofones em distribuição complementar - [d] ocorre entre sons vozeados; [t], nos demais contextos. No caso do coreano, portanto, temos duas consoantes que são mapeadas numa mesma

\footnotetext{
Para revisões em português, ver Tristão e Feitosa (2003) e Penido e Rothe-Neves (2013).

4 Contudo, não são apresentadas comparações estatísticas entre esta resposta e a resposta correspondente em uma condição de controle em que as duas categorias ocorrem com frequências semelhantes (onde não foi detectada a referida "MMN tardia").
} 
unidade no nível fonológico, ao passo que, no russo, duas unidades de representação fonológica são necessárias, uma para cada consoante. É importante notar que ambas as consoantes ocorrem nessas duas línguas sem que possam ser livremente comutadas entre si, mas apenas no russo a distinção tem consequências lexicais. Por meio de um protocolo experimental semelhante ao descrito acima, usado por Phillips et al. (2000), os autores observaram a MMNm no grupo dos falantes do russo, mas não entre os coreanos. Concluíram que a resposta obtida reflete apenas contrastes relevantes para o estabelecimento de distinções linguísticas.

Outra forma de usar a MMN na investigação de níveis abstratos de representação fonológica foi elaborada por Eulitz e Lahiri (2004) com o fim de testar predições da Teoria da Subespecificação (LAHIRI; REETZ, 2010) sobre a percepção da fala. Admitindo que a estrutura fonológica tem como unidades básicas traços distintivos e que certos traços - em particular, o traço de ponto de articulação [coronal] - podem não ser especificados no armazenamento lexical, Eulitz e Lahiri buscavam evidências de manifestações neurais de representações fonológicas subespecificadas (ou seja, representações em que algum traço que caracteriza o som correspondente não é caracterizado no léxico). Para isto, foram comparadas respostas de falantes do alemão em duas condições experimentais: (a) em que os estímulos desviante e padrão são, respectivamente, a vogal coronal /ø/ (não especificada em ponto de articulação) e a vogal dorsal /o/ (especificada com o traço [dorsal]), e (b) em que esses papéis são invertidos (desviante /o/; padrão $/ \varnothing /$ ). Essas vogais, como se vê, diferem apenas em seus pontos de articulação. A hipótese testada é a de que uma MMNm menos pronunciada seria produzida quando o estímulo padrão fosse a vogal subespecificada /ø/. Isto porque, de acordo com a tese da subespecificação, o vestígio de memória sensorial produzido pela repetição do estímulo padrão representaria regularidades menos específicas e de maneira menos precisa no caso de /ø/ em comparação com /o/, pois não incluiria o traço de ponto de articulação. Assim, o estímulo desviante não violaria nenhuma regularidade no nível dos traços fonológicos quando o estímulo padrão fosse $/ \varnothing /$, mas sim quando o estímulo padrão fosse /o/. A hipótese foi corroborada 5 .

Resultados convergentes foram obtidos em uma série de investigações posteriores comparando os traços [coronal] e [dorsal] em vogais (CORNELL; LAHIRI; EULITZ, 2011) e consoantes (SCHARINGER; BENDIXEN et al., 2012; CORNELL; LAHIRI; EULITZ, 2013) alemãs inseridas em palavras e logatomas, e também comparando consoantes plosivas e nasais do alemão (CORNELL et al., 2013) e vogais baixas e médias do inglês - as últimas consideradas como não especificadas em altura (SCHARINGER; MONAHAN; IDSARDI, 2012). Em estudos envolvendo os traços [coronal] e [labial] em consoantes, verificam-se resultados concordantes (WALTER; HACQUARD, 2004) e discordantes (SCHARINGER; MERICKEL et al., 2011) com os anteriores.

\subsection{Para além do segmento}

Reflexos da organização sonora das línguas nas respostas evocadas auditivas são também verificados no nível das combinações de segmentos fônicos para a formação de unidades maiores como sílabas, morfemas e palavras. Em primeiro lugar, verifica-se que a MMN ocorre com maiores amplitudes em resposta a palavras em comparação a logatomas (PULVERMÜLLER et al., 2001; PULVERMÜLLER et al., 2004) e a palavras mais frequentes em comparação a palavras menos frequentes (ALEXANDROV et al., 2011). Há ainda evidências de efeitos associados à frequência de ocorrência de padrões fonotáticos na língua (BONTE et al., 2005).

Em um estudo a respeito de restrições fonotáticas sobre a forma da sílaba, Dehaene-Lambertz, Dupoux e Gout (2000) compararam respostas de falantes do francês e do japonês a logatomas dissílabos com as formas VCCV (como "ebzo" e "igmo") e VCuCV (como "ebuzo" e "igumo"). No japonês, mas não no francês, são proibidas consoantes não nasais em posição de coda silábica, como bem ilustram casos de empréstimo lexical em que uma vogal /u/ ou /o/ é inserida de modo a sanar as violações da referida restrição - como, por exemplo, "kissu" (do inglês "kiss") e "karuta" (do português "carta"). Os falantes do francês, mas não os falantes do japonês, apresentaram uma resposta semelhante à MMN associada à detecção de estímulos como "ebuzo" precedidos de séries repetitivas de estímulos como "ebzo", mostrando a violação da expectativa. Isso sugere que os falantes do japonês formam, durante a percepção, representações equivalentes em resposta a sequências $\mathrm{VCuCV}$ e VCCV - presumivelmente pela inserção de uma vogal epentética no segundo caso.

Steinberg, Truckenbrodt e Jacobsen (2010a, 2010b) apresentam evidências de que violações de uma restrição fonotática do alemão ${ }^{6}$ produzem um aumento na MMN ou uma deflexão negativa adicional mais tardia (entre 250 e $400 \mathrm{~ms}$ ) em respostas evocadas por sílabas apresentadas como estímulos desviantes. Posteriormente (STEINBERG; TRUCKENBRODT; JACOBSEN, 2011),

\footnotetext{
O estudo incluía ainda condições de controle experimental em que foram apresentadas as vogais /e/ e/ø/, ambas coronais.

6 Trata-se de uma restrição violada por sequências compostas por uma vogal e uma fricativa discordantes nas especificações de traços relativos à posterioridade do ponto de articulação ([dorsal] ou [posterior]).
} 
foi obtida uma MMN por meio de um paradigma oddball em que o estímulo desviante variava entre diferentes sílabas mal formadas (que violavam aquela restrição) e o estímulo padrão entre diferentes sílabas bem formadas.

\section{Concluindo}

Como se viu, a MMN é uma resposta eletrofisiológica do córtex auditivo causada pela incongruência entre um estímulo desviante de uma série de outros estímulos. Por isso, tem sido utilizada para investigar como a percepção humana responde pré-atencionalmente ao tamanho e, principalmente, à natureza (acústica, fônica, fonotática) do "desvio" quando os estímulos são sons da fala ou não. A principal conclusão da literatura que aqui revisamos é a de que a MMN pode ser efeito de se ter produzido quaisquer categorias para agrupar estímulos auditivos, verbais ou não. Assim, a MMN, que é uma resposta a diferenças acústicas, é exagerada quando tais diferenças são usadas funcionalmente, seja numa língua, seja em qualquer outro processo em que se aprendam categorias.

Tomados em conjunto, esses estudos apontam para um processamento inespecífico para sons da fala, ao menos no nível de processamento cortical em que a MMN é gerada. O córtex auditivo humano aparece dotado de uma capacidade de categorizar e, usando-a, forma unidades cada vez mais abstratas: extrai informações linguisticamente irrelevantes e amplia diferenças acusticamente semelhantes para torná-las funcionalmente úteis, não apenas quanto a estímulos isolados, mas também quando percebidos em sequências lineares. Os resultados são consistentes com a distinção entre um nível de representação mais superficial, rico em detalhamento fonético e psicoacústico, e um nível mais abstrato, estabelecido pela fonologia da língua embora as evidências para as representações fonológicas sejam, naturalmente, mais frágeis, por força, talvez, da escassez de trabalhos que abordam relações entre medidas eletrofisiológicas e consequências de hipóteses de teorias fonológicas.

Para a teorização linguística, o que podemos aprender disso - relativamente à questão que motivou o artigo - pode ser resumido em dois aspectos. Em primeiro lugar, essa capacidade "categorizadora" no nível do córtex auditivo parece estar para a percepção como as estruturas envolvidas na articulação estão para a produção da fala: não foram criadas para isso (servem para mastigar e engolir), mas são usadas para a linguagem. Em segundo lugar, o uso que delas se faz induz o processo de formar categorias num nível muito básico, ainda antes que se possa percebê-lo conscientemente. Como isso é feito nos seres humanos ainda é uma questão a ser respondida.

\section{Referências}

ALEXANDROV, Alexander A.; BORICHEVA, Daria O.; PULVERMÜLLER, Friedemann; SHTYROV, Yuri. Strength of word-specific neural memory traces assessed electrophysiologically. PloS One, v. 6, n. 8, e22999, 2011.

AULANKO, Reijo; HARI, Riita; LOUNASMAA, Olli V. et al. Phonetic invariance in the human auditory cortex. Neuroreport, v. 4, n. 12, p. 1356-1358, Sept. 1993.

BOERSMA, Paul. Functional phonology: formalizing the interactions between articulatory and perceptual drives. 1998. 493 f. [Thesis] - Faculteit der Geesteswetenschappen, Universiteit van Amsterdam, Amsterdam, 1998.

BOERSMA, Paul; HAMANN, Silke. The evolution of auditory dispersion in bidirectional constraint grammars. Phonology, v. 25, n. 2, p. 217-270. Aug. 2008.

BOERSMA, Paul.; HAMANN, Silke. Phonology in perception. Berlin: Walter de Gruyter. 2009

BONTE, Milene L.; MITTERER, Holger;ZELLAGUI, Nadia etal. Auditory cortical tuning to statistical regularities in phonology. Clinical Neurophysiology, v. 116, n. 12, p. 2765-2774, Dec. 2005.

BUNGE, Mario. La investigacion cientifica: su estrategia y su filosofia. Barcelona: Ariel, 1976.

CHEOUR, Marie; CEPONIENE, Rita; LEHTOKOSKI, Anne et al. 1998. Development of language-specific phoneme representations in the infant brain. Nature Neuroscience, v. 1, n. 5, p. 351-353, Sept. 1998.

CHOMSKY, Noam; HALLE, Morris. The sound pattern of English. New York: Harper \& Row, 1968.

CORNELL, Sonia A.; LAHIRI, Aditi; EULITZ, Carsten. 'What you encode is not necessarily what you store': evidence for sparse feature representations from mismatch negativity. Brain Research, v. 1394, p. 79-89, June 2011.

CORNELL, Sonia A.; LAHIRI, Aditi; EULITZ, Carsten. Inequality across consonantal contrasts in speech perception: Evidence from mismatch negativity. Journal of Experimental Psychology: Human Perception and Performance, v. 38, n. 3, p. 752-772, June 2013.

DEGUCHI, Chizuru; CHOBERT, Julie; BRUNELLIÈRE, Angèle et al. 2010. Pre-attentive and attentive processing of french vowels. Brain Research. v. 1366, p. 149-161, Dec. 2010.

DEHAENE-LAMBERTZ, Ghislaine. Electrophysiological correlates of categorical phoneme perception in adults. Neuroreport, v. 8, n. 4, p. 919-924, Mar. 1997.

DEHAENE-LAMBERTZ, Ghislaine; DUPOUX, Emmanuel; GOUT, A. Electrophysiological correlates of phonological processing: a cross-linguistic study. Journal of Cognitive Neuroscience, v. 12, n. 4, p. 635-647, July 2000.

DEHAENE-LAMBERTZ, Ghislaine; PALLIER, Christophe; SERNICLAES, Willy et al. Neural correlates of switching from auditory to speech perception. NeuroImage, v. 24 n. 1, p. 21-33, Jan. 2005

DIESCH, Eugen; EULITZ, Carsten; HAMPSON, Scott; ROSS, Bernard. The neurotopography of vowels as mirrored by evoked magnetic field measurements. Brain and Language, v. 53, n. 2, p. 143-168, May 1996. 
EULITZ, Carsten; LAHIRI, Aditi. Neurobiological evidence for abstract phonological representations in the mental lexicon during speech recognition. Journal of Cognitive Neuroscience, v. 16 , n. 4 , p. 577-583, May 2004.

FLEMMING, E. Auditory representations in phonology. New York: Routledge, 2002.

FLEMMING, Edward. Speech perception and phonological contrast. In: PISONI, David B.; REMEZ, Robert E. (Ed.). The handbook of speech perception. Malden: Blackwell Publishing, 2008. p. 156-181.

GARAGNANI, Max; PULVERMÜLLER, Friedemann. From sounds to words: a neurocomputational model of adaptation, inhibition and memory processes in auditory change detection. NeuroImage, v. 54, n. 1, p. 170-181, Jan. 2011.

GERRITS, Ellen; SCHOUTEN, M. E. H. Categorical perception depends on the discrimination task. Perception \& Psychophysics, v. 66, n. 3, p. 363-376, Apr. 2004.

HILL, Penelope R.; MCARTHUR, Genevieve M.; BISHOP, Dorothy V. M. Phonological categorization of vowels: a mismatch negativity study. Neuroreport, v. 15, n. 14, p. 21952199, Oct. 2004

JÄÄSKELÄINEN, Iiro P.; AHVENINEN, Jyrki; BONMASSAR, Giorgio et al. Human posterior auditory cortex gates novel sounds to consciousness. Proceedings of the National Academy of Sciences of the United States of America, v. 102, n. 17, p. 6809-6814, Apr. 2004.

JACOBSEN, Thomas; SCHRÖGER, Erich; ALTER, Kai. Pre-attentive perception of vowel phonemes from variable speech stimuli. Psychophysiology, v. 41, n. 4, p. 654-659, July 2004

KAZANINA, Nina; PHILLIPS, Colin; IDSARDI, William. The influence of meaning on the perception of speech sounds. Proceedings of the National Academy of Sciences of the United States of America, v. 103, n. 30, p. 11381-11386, July 2006.

KIRCHNER, Robert Martin. An Effort Based Approach to Consonant Lenition. 1998. 340 f. [Thesis] - University of California, Los Angeles, 1998.

KIRMSE, Ursula; YLINEN, Sari; TERVANIEMI, Mari et al. Modulation of the mismatch negativity (MMN) to vowel duration changes in native speakers of finnish and german as a result of language experience. International Journal of Psychophysiology, v. 67, n. 2, p. 131-143, Feb. 2008.

KLATT, Dennis. H. Review of selected models of speech perception. In: MARSLEN-WILSON, William (Ed.). Lexical representation and process. Cambridge: MIT Press, 1989. p. $169-226$.

KUHL, Patricia K.; CONBOY, Barbara T.; COFFEY-CORINA, Sharon et al. Phonetic learning as a pathway to language: new data and native language magnet theory expanded (NLM-e). Philosophical Transactions of the Royal Society of London. Series B, Biological Sciences, v. 363, n. 1493, p. 979-1000, Mar. 2008

KUHL, Patricia K.; STEVENS, Erica; HAYASHI, Akiko et al. Infants show a facilitation effect for native language phonetic perception between 6 and 12 months. Developmental Science, v. 9, n. 2, p. F13-F21, Mar. 2006.
LAHIRI, Aditi; REETZ, Henning. Distinctive features: phonological underspecification in representation and processing. Journal of Phonetics, v. 38, n. 1, p. 44-59, Jan. 2010.

LAUFER, Ilan; NEGISHI, Michiro; RAJEEVAN, Nallakandi et al. Sensory and cognitive mechanisms of change detection in the context of speech. Brain Structure \& Function, v. 212, n. 5, p. 427-442, fev. 2008 .

LAUFER, Ilan; NEGISHI, Michiro; CONSTABLE, R. Todd. Comparator and non-comparator mechanisms of change detection in the context of speech - an ERP study. NeuroImage, v. 44, n. 2, p. 546-562, Jan. 2009.

LIBERMAN, Alvin M.; COOPER, Franklin S.; SHANKWEILER, D. P.; STUDDERT-KENNEDY, Michael. Perception of the speech code. Psychological Review, v. 74, n. 6, p. 431461, Nov. 1967.

LIU, Ran; HOLT, Lori L. Neural changes associated with nonspeech auditory category learning parallel those of speech category acquisition. Journal of Cognitive Neuroscience, v. 23, n. 3, p. 683-698, Mar. 2011.

LÜTKENHÖNER, Bernd; POEPPEL, David. From tones to speech: magnetoencephalographic studies. In: WINER, Jeffery A.; SCHREINER, Christoph E. (Ed.). The Auditory Cortex. New York: Springer, 2011. p. 597-615.

MAISTE, Anita S.; Andrew S. WIENS; Melvyn J. HUNT et al. Event-related potentials and the categorical perception of speech sounds. Ear and Hearing, v. 16, n. 1, 68-90, Feb. 1995.

MÄKELÄ, Anna Mari; ALKU, Paavo; TIITINEN, Hannu. The auditory $\mathrm{N} 1 \mathrm{~m}$ reveals the left-hemispheric representation of vowel identity in humans. Neuroscience Letters, v. 353, n. 2, p. 11-114, Dec. 2003.

NÄÄTÄNEN, Risto. The role of attention in auditory information processing as revealed by event-related potentials and other brain measures of cognitive function. Behavioral and Brain Sciences, v. 13, n. 2, p. 201-288, June 1990.

NÄÄTÄNEN, Risto; Teija KUJALA; István WINKLER. Auditory processing that leads to conscious perception: a unique window to central auditory processing opened by the mismatch negativity and related responses. Psychophysiology, v. 48, n. 1, p. 4-22, Jan. 2011.

NÄÄTÄNEN, Risto; Anne LEHTOKOSKI; Mietta LENNES et al. Language-specific phoneme representations revealed by electric and magnetic brain responses. Nature, v. 385 n. 6615 , p. 432-434, Jan. 1997.

NÄÄTÄNEN, Risto; PAAVILAINEN, Petri; RINNE, Teemu; ALHO, Kimmo. The mismatch negativity ( $\mathrm{mmn})$ in basic research of central auditory processing: a review. Clinical Neurophysiology, v. 118, n. 12, p. 2544-2590, Dec. 2007.

NÄÄTÄNEN, Risto; WINKLER, István. The concept of auditory stimulus representation in cognitive neuroscience. Psychological Bulletin, v. 125, n. 6, p. 826-859, 1999.

NENONEN, Sari; SHESTAKOVA, Anna; HUOTILAINEN, Minna; NÄ̈̈TÄNEN, Risto. Linguistic relevance of duration within the native language determines the accuracy of speechsound duration processing. Cognitive Brain Research, v. 16, n. 3, p. 492-495, May 2003. 
OBLESER, Jonas; ELBERT, Thomas; LAHIRI, Aditi; EULITZ, Carsten. Cortical representation of vowels reflects acoustic dissimilarity determined by formant frequencies. Brain Research. Cognitive Brain Research, v. 15, n. 3, p. 207-213, Feb. 2003.

OBLESER, Jonas; LAHIRI, Aditi; EULITZ, Carsten. Auditoryevoked magnetic field codes place of articulation in timing and topography around 100 milliseconds post syllable onset. NeuroImage, v. 20, n. 3, p. 1839-1847, Nov. 2003.

OBLESER, Jonas; LAHIRI, Aditi; EULITZ, Carsten. Magnetic brain response mirrors extraction of phonological features from spoken vowels. Journal of Cognitive Neuroscience, v. 16, n. 1, p. 31-39, Jan. 2004.

OBLESER, Jonas; SCOTT, Sophie K.; EULITZ, Carsten. Now you hear it, now you don't: transient traces of consonants and their nonspeech analogues in the human brain. Cerebral Cortex, v. 16, n. 8, p. 1069-1076, Aug. 2006.

OPITZ, Bertram; SCHRÖGER, Erich; CRAMON, D. Yes von. Sensory and cognitive mechanisms for preattentive change detection in auditory cortex. The European Journal of Neuroscience, v. 21, n. 2, p. 531-535, Jan. 2005.

PADGETT, Jaye. Perceptual Distance of Contrast: Vowel Height and Nasality. In: KATAYAMA, Motoko; KARVONEN, Dan (Ed.). Phonology at Santa Cruz, Santa Cruz: Linguistic Research Center, 1997. v. 5. p. 63-78.

PENIDO, Fabiana A.; ROTHE-NEVES, Rui. Percepção da fala em desenvolvimento: uma retrospectiva. Verba Volant, v. 4, n. 1, p. 117-140, Jan./June 2013.

PHILLIPS, Colin. Levels of representation in the electrophysiology of speech perception. Cognitive Science, v. 25, n. 5, p. 711-731, Sept. 2001.

PHILLIPS, Colin; PELLATHY, Thomas; MARANTZ, Alec et al. Auditory cortex accesses phonological categories: an meg mismatch study. Journal of Cognitive Neuroscience, v. 12, n. 6 , p. $1038-1055,2000$

PRINCE, Alan; SMOLENSKY, Paul. Optimality theory: constraint interaction in generative grammar. Technic written report CU-CS-696-93, Department of Computer Science, University of Colorado at Boulder, and Technic written report TR-2, Rutgers Center for Cognitive Science, Rutgers University, New Brunswick, NJ. 1993.

PULVERMÜLLER, Friedemann; KUJALA, Teija; SHTYROV, Yuri et al. Memory traces for words as revealed by the mismatch negativity. NeuroImage, v. 14, n. 3, p. 607-616, Sept. 2001.

PULVERMÜLLER, Friedemann; SHTYROV, Yuri; KUJALA, Teija; NÄÄTÄNEN, Risto. Word-specific cortical activity as revealed by the mismatch negativity. Psychophysiology, v. 41, n. 1, p. 106-112, Jan. 2004

ROTHE-NEVES, Rui. Notas sobre o método na Neurolinguística. In: MENDES; E. A. M.; OLIVEIRA, P. M.; BENNIBLER, V. Onovo milênio: interfaces linguísticas e literárias. Belo Horizonte: Faculdade de Letras da UFMG, 2001. p. 189-195.

SAVELA, Janne; KUJALA, Teija; TUOMAINEN, Jyrki et al. The mismatch negativity and reaction time as indices of the perceptual distance between the corresponding vowels of two related languages. Brain Research. Cognitive Brain Research, v. 16, n. 2, p. 250-256, Apr. 2003.
SCHARINGER, Mathias; BENDIXEN, Alexandra; TRUJILLO-BARRETO, Nelson J.; OBLESER, Jonas. A sparse neural code for some speech sounds but not for others. PloS One, v. 7, n. 7, e40953, July 2012.

SCHARINGER, Mathias; IDSARDI, William J.; POE, Samantha. A comprehensive three-dimensional cortical map of vowel space. Journal of Cognitive Neuroscience, v. 23, n. 12, p. 3972-3982, Dec. 2011.

SCHARINGER, Mathias; MERICKEL, Jennifer; Riley, Joshua; IDSARDI, William J. Neuromagnetic evidence for a featural distinction of english consonants: sensor- and source-space data. Brain and Language, v. 116, n. 2, p. 71-82, Feb. 2011.

SCHARINGER, Mathias; MONAHAN, Philip J; IDSARDI, William J. Asymmetries in the processing of vowel height. Journal of Speech, Language, and Hearing Research, v. 55, n. 3, p. 903-918, June 2012.

SCHOUTEN, Bert; GERRITS, Ellen; HESSEN, Arjan van. The end of categorical perception as we know it. Speech Communication, v. 41, n. 1, p. 71-80, Aug. 2003.

SHARMA, Anu; DORMAN, Michael F. Cortical auditory evoked potential correlates of categorical perception of voiceonset time. The Journal of the Acoustical Society of America, v. 106, n. 2, p. 1078-1083, Aug. 1999.

SHARMA, Anu; DORMAN, Michael F. Neurophysiologic correlates of cross-language phonetic perception. The Journal of the Acoustical Society of America, v. 107, n. 5 Pt 1, p. $2697-$ 2703, May 2000.

SHARMA, Anu; KRAUS, Nina; MCGEE, Therese et al. Acoustic versus phonetic representation of speech as reflected by the mismatch negativity event-related potential. Electroencephalography and Clinical Neurophysiology, v. 88, n. 1, p. 64-71, Jan./Feb. 1993.

SHESTAKOVA, Anna; BRATTICO, Elvira; HUOTILAINEN, Minna et al. Abstract phoneme representations in the left temporal cortex: magnetic mismatch negativity study. Neuroreport, v. 13, n. 14, p. 1813-1816, Oct. 2002.

SHESTAKOVA, Anna; BRATTICO, Elvira; SOLOVIEV, Alexei et al. Orderly cortical representation of vowel categories presented by multiple exemplars. Brain Research. Cognitive Brain Research, v. 21, n. 3, p. 342-350, Nov. 2004

SILVA, Daniel M. R.; ROTHE-NEVES, Rui. Um estudo experimental sobre a percepção do contraste entre as vogais médias posteriores do português brasileiro. DELTA, v. 25, n. 2, p. 319-345. 2009.

STEINBERG, Johanna; TRUCKENBRODT, Hubert; JACOBSEN, Thomas. Preattentive phonotactic processing as indexed by the mismatch negativity. Journal of Cognitive Neuroscience, v. 22, n. 10, p. 2174-2185, Oct. 2010.

STEINBERG, Johanna; TRUCKENBRODT, Hubert; JACOBSEN, Thomas. Activation and application of an obligatory phonotactic constraint in german during automatic speech processing is revealed by human event-related potentials. International Journal of Psychophysiology, v. 77, n. 1, p. 13-20, July 2010 .

STEINBERG, Johanna; TRUCKENBRODT, Hubert; Thomas JACOBSEN. Phonotactic constraint violations in German grammar are detected automatically in auditory 
speech processing: a human event-related potentials study. Psychophysiology, v. 48, n. 9, p. 1208-1216, Sept. 2011.

STEINSCHNEIDER, Mitchell; LIÉGEOIS-CHAUVEL, Catherine; BRUGGE, John F. Auditory evoked potentials and their utility in the assessment of complex sound processing. In: WINER, Jeffery A.; SCHREINER, Christoph E. (Ed.). The Auditory Cortex. New York: Springer, 2011. p. 535-559.

TERVANIEMI, Mari; JACOBSEN, Thomas; RÖTTGER, Stefan et al. Selective tuning of cortical sound-feature processing by language experience. The European Journal of Neuroscience, v. 23, n. 9, p. 2538-2541, May 2006.

TRISTÃO, Rosana Maria; FEITOSA, Maria Ângela G. Percepção da fala em bebês no primeiro ano de vida. Estudos de Psicologia (Natal), v. 8, n. 3, p. 459-467, dez. 2003.

TUOMAINEN, Jyrki; SAVELA, Janne; OBLESER, Jonas; AALTONEN, Olli. Attention modulates the use of spectral attributes in vowel discrimination: behavioral and event-related potential evidence. Brain Research, v. 1490, p. 170-183, Jan. 2013.
WALTER, Mary Ann; VALENTINE Hacquard. Meg evidence for phonological underspecification. In: INTERNATIONAL CONFERENCE ON BIOMAGNETISM, 14 ${ }^{\text {th }}, 2004$, Boston. Proceedings... Boston, 2004. p. 292-293.

WERKER, Janet F.; TEES, Richard C. Speech perception as a window for understanding plasticity and commitment in language systems of the brain. Developmental Psychobiology, v. 46, n. 3, p. 233-251, Apr. 2005.

WINKLER, István; LEHTOKOSKI, Anne; ALKU, Paavo et al. Pre-attentive detection of vowel contrasts utilizes both phonetic and auditory memory representations. Cognitive Brain Research, v. 7, n. 3, p. 357-369, Sept. 1999.

ZHANG, Yang; KUHL, Patricia K.; IMADA, Toshiaki et al. Effects of language experience: neural commitment to languagespecific auditory patterns. NeuroImage, v. 26, n. 3, p. 703-720, July 2005.

Recebido: 27 de agosto de 2013

Aprovado: 20 de dezembro de 2014

Contato: silvadmr@gmail.com; rothe.neves@gmail.com 